ARTICLE

DOI: $10.1038 / s 41467-017-02336-z$

\title{
Broadband impedance match to two-dimensional materials in the terahertz domain
}

Phi H.Q. Pham', Weidong Zhang ${ }^{2}$, Nhi V. Quach', Jinfeng Li, Weiwei Zhou', Dominic Scarmardo', Elliott R. Brown ${ }^{2} \&$ Peter J. Burke ${ }^{1}$

The coupling of an electromagnetic plane wave to a thin conductor depends on the sheet conductance of the material: a poor conductor interacts weakly with the incoming light, allowing the majority of the radiation to pass; a good conductor also does not absorb, reflecting the wave almost entirely. For suspended films, the transition from transmitter to reflector occurs when the sheet resistance is approximately the characteristic impedance of free space $\left(Z_{0}=377 \Omega\right)$. Near this point, the interaction is maximized, and the conductor absorbs strongly. Here we show that monolayer graphene, a tunable conductor, can be electrically modified to reach this transition, thereby achieving the maximum absorptive coupling across a broad range of frequencies in terahertz $(\mathrm{THz})$ band. This property to be transparent or absorbing of an electromagnetic wave based on tunable electronic properties (rather than geometric structure) is expected to have numerous applications in $\mathrm{mm}$ wave and $\mathrm{THz}$ components and systems.

\footnotetext{
${ }^{1}$ Department of Electrical Engineering and Computer Science, University of California, Irvine, CA 92697, USA. ${ }^{2}$ Department of Physics and Electrical Engineering, Wright State University, Dayton, $\mathrm{OH}$ 45435, USA. Correspondence and requests for materials should be addressed to

P.J.B. (email: pburke@uci.edu)
} 
T wo-dimensional materials provide a class of atomically thin conductors ${ }^{1-3}$ which can be synthesized with area much larger than the electromagnetic (EM) wavelength in the $\mathrm{mm}$ wave and $\mathrm{THz}$ bands, creating opportunities to control EM beams without the need for nanoscale patterning that is typically required in the optical ${ }^{4,5}$. To date, unpatterned monolayer graphene devices have operated exclusively in the weakly coupled, highly transmissive regime $e^{6-18}$.

Here, by deliberate engineering of the sheet conductance using large-domain graphene films in combination with chemically modified substrates (to decrease interface scattering), as well as chemical and electrical doping, we fabricate monolayer graphene devices with sheet resistance crossing the characteristic impedance of free space, $377 \Omega / \square$ Achieving this, we show that monolayer graphene can be tuned to behave as a strong absorber over a broadband frequency range, approaching the theoretical impedance at which absorption is maximized ${ }^{19,20}$. In free space, the maximum absorption is $50 \%$, whereas on a substrate it can be greater than $90 \%$ depending on the direction of normal incidence, discussed in detail below. Regardless of the case (free space or on dielectric), the absorption is much larger than the $2.3 \%$ optical value $^{21}$, and thus represents a milestone of coupling electromagnetic waves to an atomically thin nanomaterial. This is measured over an extremely broad range, from $\mathrm{mm}$ wave to $\mathrm{THz}$ frequencies. Strong EM absorption using a single atomic layer exemplifies the fundamental relationship between nanoscale electronics and classical electromagnetism.

\section{Results}

THz conductance. The zero-gap band structure of graphene leads to a non-trivial frequency-dependent AC conductance, which governs the absorption of the EM radiation. As depicted in Fig. 1a, at optical frequencies the sheet conductance of graphene is dominated by interband transitions, and exhibits a universal value of $G_{\mathrm{g}}=(\pi / 4) \cdot G_{0}=\left(e^{2} / 4 \hbar\right)^{21}$, where $G_{0}$ is the conductance quantum. This results in its low absorbance, approximated by the product of $G_{\mathrm{g}}$ and the characteristic impedance of free-space, $Z_{0}=377 \Omega$. This product can be written $\pi \cdot\left(e^{2} / \hbar c\right) \equiv \pi \cdot \alpha$, where $\alpha$ is the fine structure $=1 / 137$. Thus, only $\approx 2.3 \%$ of the light is absorbed, and the majority of the light is transmitted.

In contrast, at frequencies lower than $\sim 2 E_{\mathrm{F}} / h$ (where $E_{\mathrm{F}}$ is the Fermi energy), as is the case in the $\mathrm{THz}$ range, the photon energy is too low to excite electron-hole pairs, and the electromagnetic sheet conductance is expected to follow the DC sheet conductance 22,23 . This should occur for frequencies up to $\sim 1 / \tau$, where $\tau$ is the scattering time, at which point the conductance is expected to undergo a Drude-like roll-off ${ }^{6-9}$, dropping towards the low optical value, (Fig. 1a). The AC conductance can be adjusted by engineering $E_{\mathrm{F}}$ and $\tau$ according to the equations:

$$
\begin{aligned}
& G_{\mathrm{DC}}=\frac{e^{2}}{4 \hbar} \frac{4}{\pi} \frac{E_{\mathrm{F}} \tau}{\hbar} \\
& G(\omega)=\frac{G_{\mathrm{DC}}}{1+i \omega \tau}
\end{aligned}
$$

This creates the opportunity to adjust the $\mathrm{THz}$ conductance into the regime where the majority of the incident light is absorbed $(A>R, T)$. This prospect is indicated schematically within the $\mathrm{THz}$ frequency range in Fig. $1 \mathrm{~b}$, where hypothetical conductance curves for plausible $E_{\mathrm{F}}$ and $\tau$ values are plotted. Figure 1c presents "device design" charts (calculated for 100 $\mathrm{GHz}$ ), which show how combinations of $E_{\mathrm{F}}$ and $\tau$ affect the AC
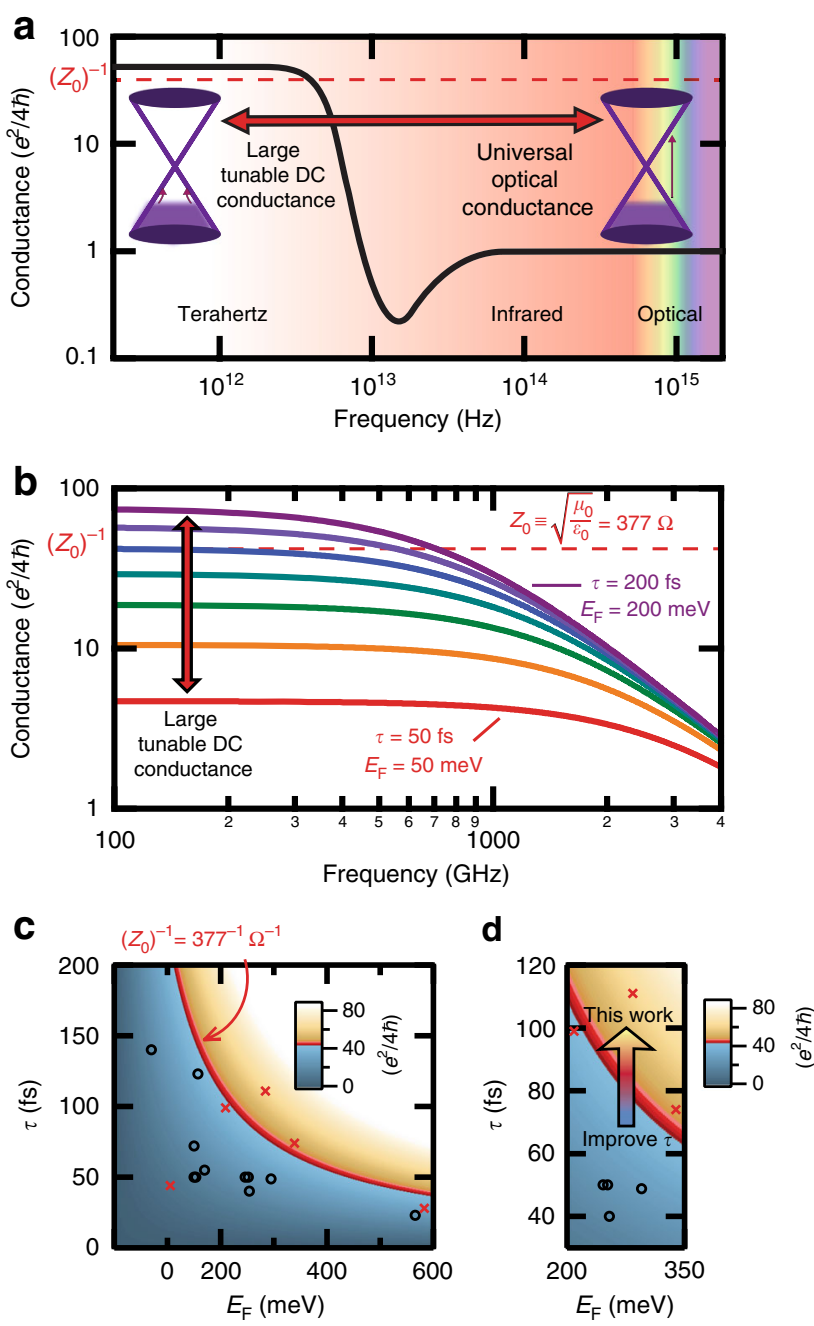

Fig. $1 \mathrm{THz}$ conductance. a Qualitative trend of frequency-dependent $\mathrm{AC}$ conductance for monolayer graphene with normalization to $e^{2} / 4 \hbar$. The optical frequency range exhibits a universal value of 1 , while in the $\mathrm{THz}$ range, the $A C$ conductance can be orders of magnitude higher. $\mathbf{b}$ Theoretical trend of $\mathrm{THz} \mathrm{AC}$ conductance for monolayer graphene plotted with a linear variation of the $E_{\mathrm{F}}$, and $\tau$. Graphene samples can cross the free space conductance value, $\left(Z_{0}\right)^{-1}$, within the $\mathrm{THz}$ regime. c Device design plot showing the graphene sheet conductance (at $100 \mathrm{GHz}$ ) in units of $\left(e^{2} / 4 \hbar\right)$ for changes in $E_{\mathrm{F}}$ and $\tau$. The $\left(Z_{0}\right)^{-1}$ threshold is set as red on the color scale. Previous graphene $\mathrm{THz}$ device parameters are plotted as circles, whereas those from this work are denoted as $x^{\prime}$ s. $\mathbf{d}$ By decreasing the electron scattering in our devices to increase $\tau$, the $\left(Z_{0}\right)^{-1}$ threshold can be crossed into the absorption-dominating regime

conductance; the absorption-dominating regime begins when the sheet resistance $\leq 377 \Omega / \square$ (set as red on the $z$-axis color scale). Previous investigations of single-layer graphene $\mathrm{THz}$ absorption fail to surpass the $Z_{0}=377 \Omega$ threshold $^{6-18}$ (denoted as circles in Fig. 1c), even in the case where $E_{\mathrm{F}}$ was designed to be purposefully large (using heavy chemical doping 6 , 15 , or strong electrical gating $6,7,10,12,13,15)$. Instead, as demonstrated in this work and emphasized as an arrow in Fig. 1d, by carefully fabricating devices with improved $\tau$, the graphene conductance can be large (denoted as an ' $x$ ' in Fig. 1c), and the device sheet resistance can be tuned to be below the free space impedance threshold. We successfully surpass this threshold with a graphene sheet, and reach near maximum absorption. 


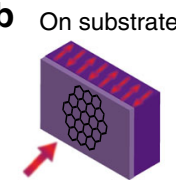

(Incident on graphene)

(Incident on substrate)

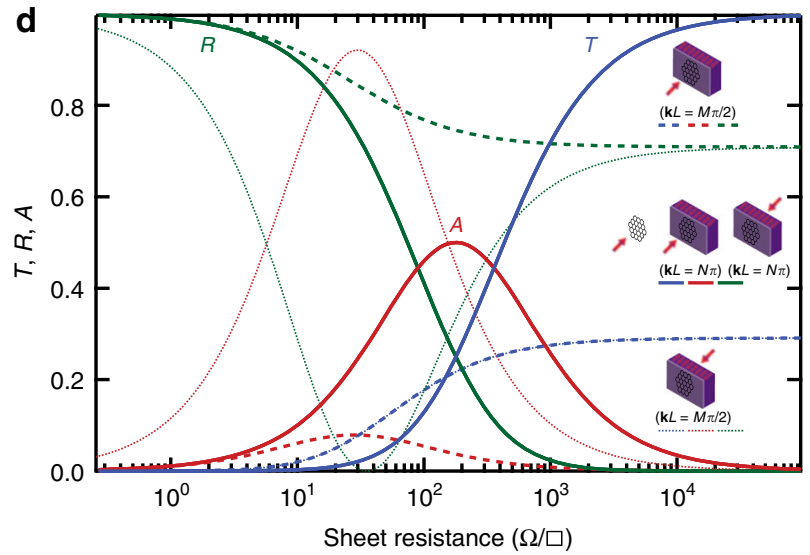

Fig. 2 Transmission, reflection and absorption. a-c A suspended film and a film on a substrate for two incidence directions were analyzed using the transmission line model. $n=3.41$ was used as the substrate index of refraction for calculation. d The suspended film/Woltersdorff equations (plotted as solid lines) for $T, R$ and $A$ vs. sheet resistance were compared to a film on a substrate. When the half-wave resonance condition is satisfied $(\mathbf{k} L=N \pi$, where $N$ is an integer), the equations for $T, R$ and $A$ reduce to exactly the Woltersdorff equations (plotted for both directions as solid lines, and are indistinguishable from the suspended film). Hence, analyzing the device at half-wave resonance provides a direct comparison of the graphene absorption on a substrate to that of a suspended film. At quarterwave resonance ( $\mathbf{k} L=M \pi / 2$, where $M$ is an odd integer), $T$ is the same for both incidence directions, but $R$ and $A$ are different for each incidence direction (plotted as the dotted lines)

Transmission, reflection and absorption regimes. Our measurement technique consists of using an ultra-broadband $\mathrm{THz}$ spectrometer, based on the photomixing of two optical lasers to generate a coherent $\mathrm{THz}$ beam $^{24}$. This system (block diagram shown in Supplementary Fig. 1) enables continuous transmission measurement from $200 \mathrm{GHz}$ to $1.2 \mathrm{THz}$ with over $60 \mathrm{~dB}$ dynamic range at the low end, and up to $40 \mathrm{~dB}$ at the high end. The graphene films are mounted on high-resistivity silicon substrates of thickness $\sim 400 \mu \mathrm{m}$, which do not absorb significantly in the $\mathrm{THz}$ region. Because of the difference in the index of refraction between the silicon substrate $(n \approx 3.4)$ and air $(n=1)$, there is an etalon effect present in the frequency-dependent transmission (even in the absence of graphene). With graphene present, we can model the effect of varying the graphene sheet resistance to calculate the net transmittance using the transmission line model (Supplementary Note 1-4), and compare these values (for a film on a substrate with incidence in both directions) to that of a suspended graphene film (Fig. 2a-c).

A plane wave can be transmitted $(T)$, reflected $(R)$ or absorbed (A). Electromagnetics states that a perfect conductor is a mirror $(T=0 ; R=1 ; A=0)$ and a perfect insulator is a transmitter $(T \leq 1$, $R \geq 0, A=0)$. What is the quantitative relationship between $T, R$, $A$ and the sheet resistance? The three are not completely independent, as $T+R+A$ must equal unity due to conservation of energy. The relationship for the transmittance, reflectance, and absorptance of a suspended thin metallic film was derived in
$1934^{19}$, and is given by,

$$
\begin{gathered}
T=\frac{4 g^{2}}{(1+2 g)^{2}} \\
R=\frac{1}{(1+2 g)^{2}} \\
A=\frac{4 g}{(1+2 g)^{2}} \\
g \equiv R / Z_{0}
\end{gathered}
$$

These expressions show the change from transmissiondominating to absorption-dominating occurring at the critical threshold of $Z_{0}=377 \Omega$. Within the absorption-dominating regime, the peak absorption occurs when the sheet resistance is equal to $Z_{0} / 2$ (Fig. $2 \mathrm{~d}$ ).

When mounted on a substrate of index of refraction, $n$, numerous considerations such as direction of normal incidence (on the graphene or substrate side first) and substrate thickness govern $T, R$ and $A$. Furthermore, interference effects from the substrate cause Fabry-Perot behavior depending on the $\mathrm{THz}$ frequency $(f)$, substrate thickness $(L)$ and index of refraction $(n)$. When incident on the graphene side first, the general analytical expressions for $T, R$ and $A$ are given by,

$$
\begin{aligned}
& T=4 Z_{0}\left|\frac{g n(n \sin (\mathbf{k} L)-i \cos (\mathbf{k} L))}{\left(1+g+g n^{2}\right) Z_{0} \sin (\mathbf{k} L)-i(1+2 g) n Z_{0} \cos (\mathbf{k} L)}\right|^{2} \\
& \frac{2 Z_{0}}{1+n^{2}-\cos (2 \mathbf{k} L)\left(n^{2}-1\right)}
\end{aligned}
$$

$$
R=\left|\frac{\left(1-g+g n^{2}\right) \sin (\mathbf{k} L)-i n \cos (\mathbf{k} L)}{\left(1+g+g n^{2}\right) \sin (\mathbf{k} L)-i(1+2 g) n \cos (\mathbf{k} L)}\right|^{2}
$$

$$
A=4 g Z_{0}^{2}\left|\frac{\sin (\mathbf{k} L)-i n \cos (\mathbf{k} L)}{\left(1+g+g n^{2}\right) Z_{0} \sin (\mathbf{k} L)-i(1+2 g) n Z_{0} \cos (\mathbf{k} L)}\right|^{2}
$$

Similar expressions for incidence on the substrate side are given in Supplementary Note 4.

For both incidence directions, when $\mathbf{k} L=N \pi$, where $\mathbf{k}$, is the wave vector, $L$ is the substrate thickness, and $N$ is integer values, or when the $\mathrm{THz}$ frequency is at half-wave resonance with the graphene-silicon etalon, which occurs at even integer multiples of $f=\frac{c}{(4 * n * L)}$, the equations for $T, R$ and $A$ reduce to exactly the same as the Woltersdorff equations ${ }^{19}$ (Eqs. 3-5). Hence, for a broadband transmittance measurement, at half-wave resonance, or at the transmittance maxima (up to a critical sheet resistance value, after which the half-wave resonance occurs at the transmittance minima, see Supplementary Note 3 and Supplementary Fig. 4e), the behavior of the graphene film can be directly compared to a suspended film, where the onset of strong absorption occurs at $Z_{0}=377 \Omega$, with maximum absorption of $50 \%$ occurring at $Z_{0} / 2$. In Fig. $2 \mathrm{~d}$ the half-wave resonance condition for $T, R$ and $A$ are indistinguishable from those of the suspended case.

In contrast, at quarter-wave resonance, when $\mathbf{k} L=M \pi / 2$, where $M$ is odd integer values, the sheet resistance-dependent 
a
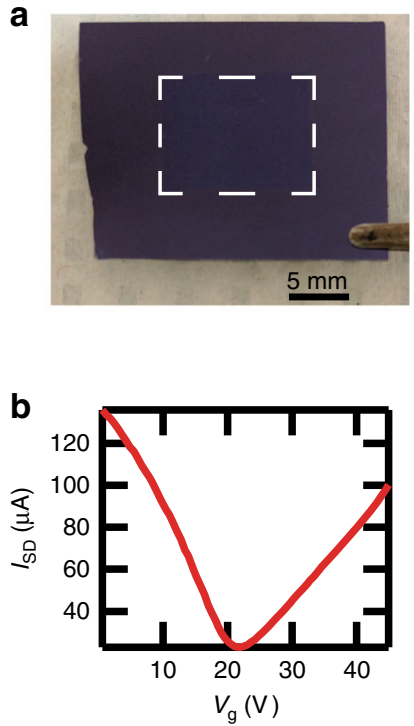

High sheet resistance

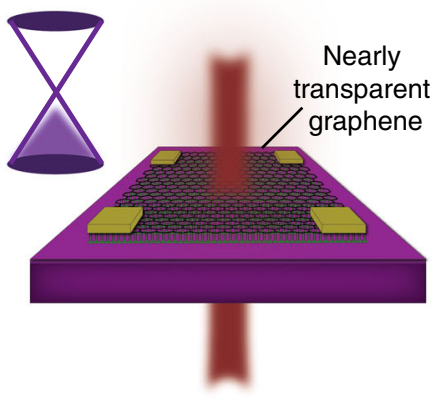

Low sheet resistance

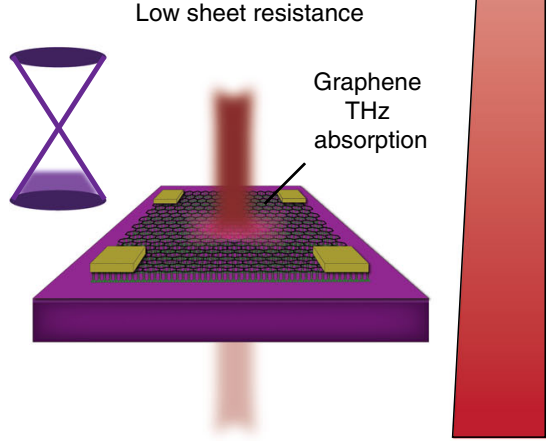

C

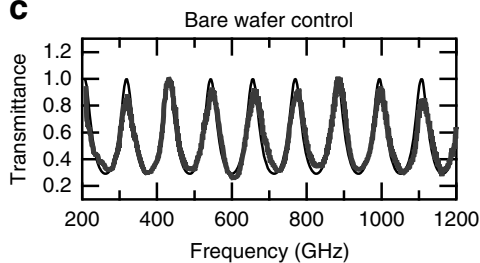

d

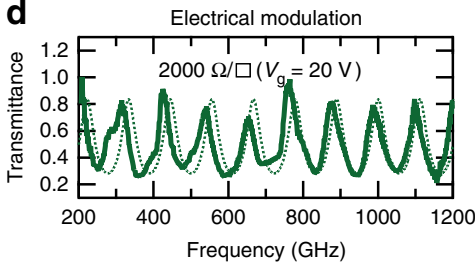

e

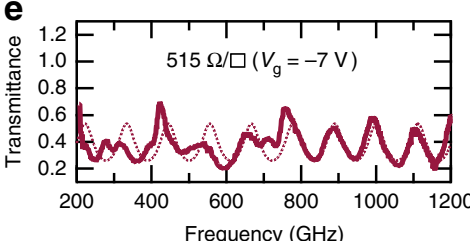

f Chemical modulation

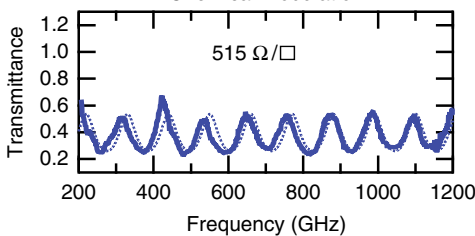

g

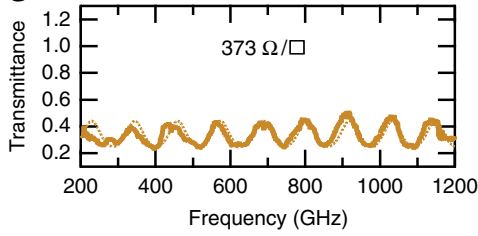

h

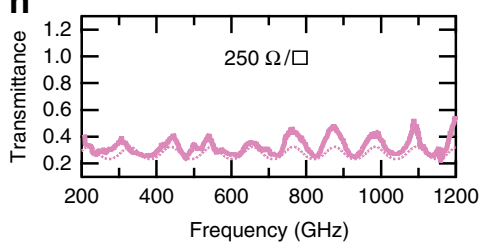

Fig. 3 Broadband impedance matching. a Optical photograph of graphene transferred onto ODTS modified oxide on high-resistivity silicon substrate. The scale bar is $\sim 5 \mathrm{~mm}$. b Source-drain current vs. gate voltage for typical graphene on ODTS SAM device. The charge neutral point is $\sim 22 \mathrm{~V}$. c Transmittance vs. frequency data acquired for a control sample (bare high-resistivity silicon substrate with no graphene). d, e Transmittance vs. frequency data for a graphene sample gated at $20 \mathrm{~V}$ and $-7 \mathrm{~V}$. f-h Transmittance vs. frequency for three devices after chemical doping to modify the zero-bias sheet resistance. The expected transmittance values assuming the DC conductance as the AC contribution (assuming negligible susceptance) are plotted as dotted lines

transmission through the graphene-silicon etalon remains low (transmission never dominates). Although the transmittance is low and equivalent for both incidence directions (graphene or substrate first), the sheet resistance-dependent reflection and absorption indeed depend on the incidence direction. When the $\mathrm{THz}$ beam is incident on the graphene side first, interference effects cause the device to behave as mostly reflecting (always reflection dominating), resulting in low absorption. On the other hand, when the $\mathrm{THz}$ beam is incident on the substrate first (with graphene on the backside), the absorption, transmission and reflection can be affectedly tuned by the sheet resistance. This allows the absorption maximum to increase to $>50 \%$ for low sheet resistance values (Supplementary Fig. 5a).

The two extreme cases (half-wave resonance and quarter-wave resonance) shows contrasting behavior, but like the suspended film, there still exists certain impedance values that determine the peak absorption, and the onset of the absorption-dominating threshold (Fig. 2d). The absorption-dominating threshold and peak absorption can shift (to even lower sheet resistance values) depending on the substrate index of refraction. We have calculated and plotted this trend for various values of the index of refraction in Supplementary Fig. 5b. Regardless of whether the 
graphene film is suspended or on a substrate, the largest sheet resistance value (most resistive) in which the film becomes absorption dominating is $377 \Omega / \square$, and hence this value signifies the doorway to strong absorption. Detailed below, we observe a decrease in the broadband transmittance as the graphene sheet resistance is tuned below $377 \Omega / \square$, signifying absorption approaching the $50 \%$ maximum for a $\mathrm{THz}$ beam incident on a graphene sheet on substrate.

Graphene device fabrication on modified substrate. Previously, researchers used bare, unpatterned graphene, and measured the $\mathrm{THz}$ resistance to be greater than $377 \Omega^{6-18}$. This includes our previous work ${ }^{9}$, where the graphene-etalon structures were fabricated using chemical vapor-deposited graphene films ${ }^{25}$ transferred $^{26}$ onto a $90 \mathrm{~nm}$ gate oxide layer on high-resistivity silicon substrates. To improve $\tau$, so as to increase the conductance of the devices, we synthesized large-domain (mm-sized) graphene films (to minimize graphene grain boundaries) ${ }^{27}$, in addition to depositing octadecyltrichlorosilane (ODTS) self-assembled monolayers (SAM) on the wafer (to decrease scattering) ${ }^{28}$ prior to graphene transfer (Supplementary Note 5). Across the measured frequency range, the SAM does not significantly affect the $\mathrm{THz}$ absorption (Supplementary Note 6). The resulting air/graphene/substrate structure shown in Fig. 3a was used for $\mathrm{THz}$ transmission measurements with the $\mathrm{THz}$ beam incident on the graphene side first. When gating the graphene to the charge neutral point $(\mathrm{CNP})$ without the use of a SAM, large voltages $(>50 \mathrm{~V})$ must be applied to the high-resistivity silicon layer. Utilization of the SAM layer provides significant improvement of the transconductance of the graphene film, and a smaller operating voltage window (Supplementary Fig. $7 \mathrm{~b}-\mathrm{c}$ ). The depletion curve shown in Fig. $3 \mathrm{~b}$ shows a CNP at $\sim 22 \mathrm{~V}$, and indicates slight $p$-doping of the graphene film. We performed a series of $\mathrm{THz}$ transmission measurements on these devices for a variety of sheet resistance values, as shown in Fig. $3 \mathrm{~d}-\mathrm{h}$.

Electrical modulation of broadband THz. In order to deconvolve the etalon effect, the broadband transmission through a bare high-resistivity silicon substrate (without graphene) is first measured as a control. The multiple interference peaks of transmission around $\approx 1.0$ (Fig. $3 \mathrm{c}$ ) confirm the low-loss nature of the bare substrate. Upon the addition of a monolayer sheet of graphene on the ODTS SAM buffer layer (and when applying a voltage on the silicon substrate near the $V_{\mathrm{CNP}}(\approx 20 \mathrm{~V})$ as shown in Fig. 3d), the graphene film behaves as a nearly transparent film (sheet resistance $\approx 2 \mathrm{k} \Omega / \square$ ), as the amplitudes of the transmission peaks approach unity. The theoretical graphene-on-Si etalon transmittance vs frequency curves, calculated for an AC admittance that is purely real (susceptance equal to zero) and equal to the measured DC conductance, are plotted as dotted lines for comparison, and generally agree with the measured values. This is clearly in the transmission-dominating regime, as the presence of the graphene has little effect on the $\mathrm{THz}$ transmission through the sample.

We next demonstrate the ability to electrically tune to the absorption-dominating regime. By applying $-7 \mathrm{~V}$, the graphene DC sheet resistance is decreased to $\approx 515 \Omega / \square$, and we observe considerably decreased maxima in the transmission peaks (Fig. 3e). Despite the fact that the gate voltage drop across the graphene film is small compared to the high-resistivity silicon substrate, consistent with the $I-V$ curve (Fig. $3 b$ ), our device still enables significant control of the single-layer graphene sheet resistance, which results in a large transmittance variation of the incident $\mathrm{THz}$ radiation. The gate-modified transmittance peak values are plotted together with the $I-V$ characteristics in
Supplementary Fig. 8a for an additional device. Gate leakage through the oxide and ODTS SAM $\left(\left[10^{-5}\right] \mathrm{A}\right)$ was observed at voltages beyond this range, and hence gate voltages were restricted to $-7 \mathrm{~V}$ to $+45 \mathrm{~V}$. Across the broadband frequency range measured, the transmittance variation at the peaks can be used to define a depth of modulation (DoM) (relevant to the use as a spatial-light modulator), defined as $\left(T_{\text {High }}-T_{\text {Low }}\right) / T_{\text {High. }}$ The DoM is substantial, with a maximum value of $\approx 52 \%$ at the $\sim 330$ $\mathrm{GHz}$ peak (Supplementary Fig. 8b). For a simple, unpatterned device geometry, consisting of only one monolayer, this is the largest transmittance variation that has been reported to date for a graphene-based modulator, to the best of our knowledge $9,10,12$, 15,17 . A $52 \%$ DoM is comparable to other patterned graphene devices such as extraordinary optical transmission structures ${ }^{29}$ and periodic arrays of graphene nanodisks ${ }^{30}$. Compared to patterned device geometries, which usually create frequency- or polarization-dependent behavior, our device structure (which does not require any lithography) allows for useful $\mathrm{THz}$ modulation over $1 \mathrm{THz}$ of frequency range with polarization independence. This result was independently confirmed in two different labs (Supplementary Note 7).

Impedance matching using chemical doping. Figure 4a shows the inferred frequency-dependent conductance varying from much less than $\left(Z_{0}\right)^{-1}$ (green curve, $V_{\mathrm{g}}=+20 \mathrm{~V}, 10 \times$ less than $\left(Z_{0}\right)$ ${ }^{-1}$ ), to approximately equal to $\left(Z_{0}\right)^{-1}$ (red curve, $V_{\mathrm{g}}=-7 \mathrm{~V}$ ), and clearly demonstrates electrical tuning from the transmission to absorption-dominating regimes. In order to achieve the necessary sheet conductance values near maximum absorption, and beyond $\left(Z_{0}\right)^{-1}$, we utilized chemical (rather than electrical) tuning. We intentionally dope graphene films to have low sheet resistance in the zero-bias state (to mitigate inconsistencies that may arise from the inhomogeneous gating using a high-resistivity substrate, and to ensure no gate leakage occurs) by using benzimidazole (BI) dissolved into the copper etching solution during graphene transfer $^{31}$ (Supplementary Note 8 ). Figure $3 \mathrm{f}-\mathrm{h}$ shows the broadband transmittance vs. frequency for three graphene films having zero-bias DC sheet resistances of 515,373 and $250 \Omega / \square$, respectively. Similar to the electrical modulation, we observe a steady decrease in the $\mathrm{THz}$ transmittance peaks. The lowest sheet resistance is clearly less than $377 \Omega$ and the trend of reduced transmission with reduced sheet resistance is observed over the entire measurement band.

Drude conductance and strong absorption. The measured transmission spectra can be used to determine the AC sheet conductance, using the bare silicon control sample as nearly lossless "calibration" (although it is noted that the transmission peaks do not necessarily remain at a fixed frequency when covered by a thin film). For devices covered by a graphene film, each transmittance peak is fitted to yield a AC admittance (real conductance and imaginary susceptance) value; the measured DC conductance and the estimated $\mathrm{THz}$ conductance are then fitted according to Eqs. 1 and 2 to estimate $E_{\mathrm{F}}$ and $\tau$ for each device (Supplementary Note 9). The calculated peak conductance values are shown in Fig. $4 \mathrm{a}$, b, with a dotted red line showing the $377 \Omega$ threshold. The predicted Drude conductance using the estimated $E_{\mathrm{F}}$ and $\tau$ is plotted as a solid line, and closely matches the measured DC value (shown on the $y$-axis). Supplementary Note 10 summarizes in a graphical representation our achievement crossing the free-space impedance threshold within the $\mathrm{mm}$ wave and $\mathrm{THz}$ frequency ranges, and compares our data to previous measurements in the literature. In this work, the frequencydomain measurement is especially broad (spanning the sub- $\mathrm{THz}$ regime), and the optical conductance and $\mathrm{THz}$ absorption of the 

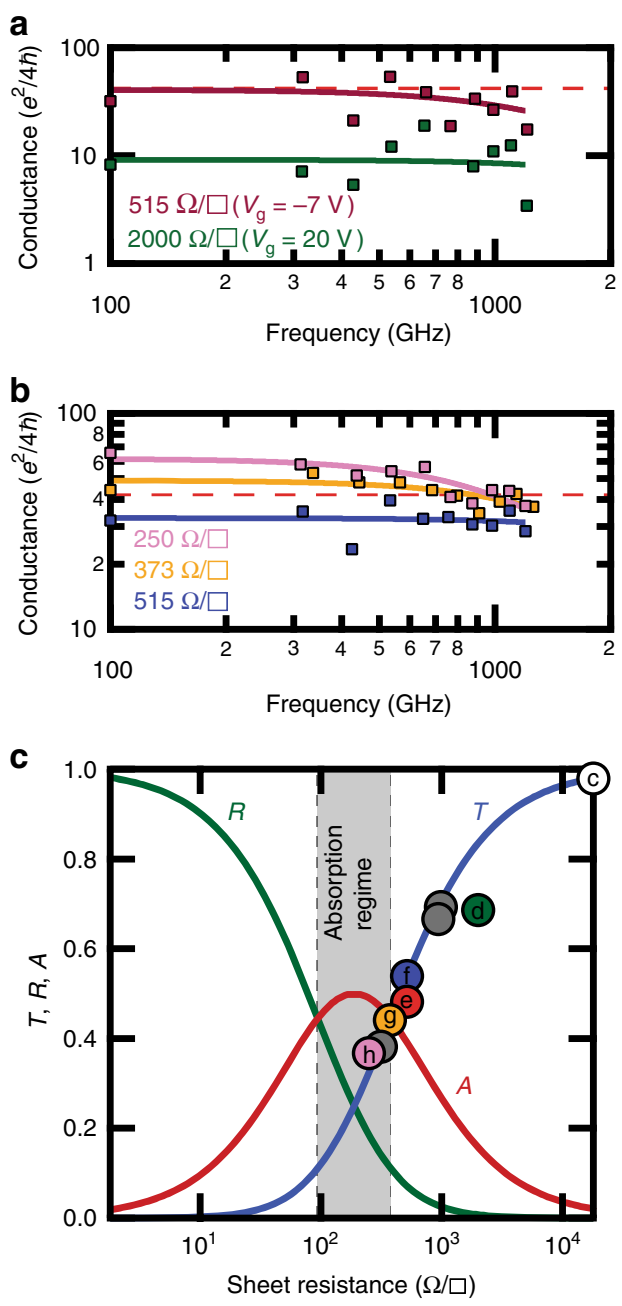

Fig. 4 Drude conductance and strong absorption. a, b The AC conductance values calculated by fitting the measured transmittance peaks for both electrically and chemically modified devices from Fig. 3 are plotted as squares. Drude model trends estimated from the frequency-dependent $A C$ conductance data are plotted as solid lines. c The measured transmittance peak values (at $\sim 655 \mathrm{GHz}$ ) are plotted as a function of sheet resistance. Colored (labeled) circles represent data from devices shown above, gray circles are from devices measured, but not displayed in Fig. 3, and the white circle represents the control sample. The theoretical transmittance, absorbance and reflectance values calculated from the half-wave resonance case for a device on a substrate are plotted as solid lines. The transmittance value becomes less than the absorbance value at sheet resistance values $<377 \Omega / \square$, marking the beginning of the absorption regime

graphene film are remarkably large for one atomic layer. Supplementary Table 1 shows the extracted Drude parameters, $E_{\mathrm{F}}$ and $\tau$, for measured devices. Notably, the values of $E_{\mathrm{F}}$ are moderate, while the values of $\tau$ can be large (>100 fs), and stress the importance of engineering $\tau$, as initially outlined in the device design plots in Fig. 1c, d. These figures clearly demonstrate our ability to tune the sheet resistance of monolayer graphene, either electrically or chemically, from well above to below the characteristic impedance of free space. We next focus on our more quantitative demonstration of the relationship between the transmittance and the sheet resistance.

We plot in Fig. 4c the predicted transmission (blue line), reflection (green line) and absorption (red line) for a graphene film as a function of sheet resistance for a film mounted on a silicon substrate, at half-wave resonance satisfying the condition $\mathbf{k} L=N \pi$. At half-wave resonance, the free-space and etalon effect give the same predicted $T, R$ and $A$ vs. sheet resistance (Fig. 2). We also show the measured transmittance peak (centered around $\sim 655 \mathrm{GHz}$ ) values (color points are from devices measured in Fig. 3, gray points are for devices measured during this work, but not displayed in Fig. 3). The $377 \Omega$ vertical dotted line indicates the characteristic impedance of free-space threshold, and marks the start of the absorption regime, where the fractional absorbance $(\sim 0.44)$ of the $\mathrm{THz}$ radiation by the graphene film becomes greater than the fractional transmittance (and reflectance $)^{19}$. Here, we have realized the crossover from the transmission regime, and have measured correspondingly small transmittance values for devices within the absorption regime. For our best single-layer device of $\approx 250 \Omega / \square$, the value of the graphene film absorbance is calculated to be $\approx 48.6 \%$ : approaching the theoretical limit of 50\% (for normal incidence on graphene side first).

\section{Discussion}

Instead of designing the single-layer sheet resistance to cross into the absorption-dominating regime, previous experiments for unpatterned graphene devices boosted low absorbance by the addition of multilayers ${ }^{32-35}$, or by suppressing the transmission channel $^{36}$ using double-pass/reflection/"Salisbury screen" geometries $^{11,14}$. Other thin film (multilayer) materials have also demonstrated remarkable control of absorption by controlling film thickness, such as niobium nitride $(\mathrm{NbN})^{37-39}$ and indium tin oxide $(\mathrm{ITO})^{40}$. Here, our experiments show that exceptionally large, near maximum, absorption can be reached in the thickness limit of one single layer of graphene.

Impedance matching to the free-space threshold using a double-pass, reflection mode geometry could achieve $100 \%$ absorption $^{34,41}$, and may further enhance the single-layer reflection $\mathrm{DoM}^{11}{ }^{42}$. Pertaining to designing devices that operate in transmission (such as the work performed here), the suspended film and Woltersdorff equations ${ }^{19}$ stand as the ideal situation for broadband absorption. Using the transmission line model for a film on a substrate, as the substrate index of refraction value approaches $n=1$, or the substrate thickness approaches, $L=0$, the amplitudes of the Fabry-Perot fringes decrease, and the widths of the fringes increase (as calculated values converge toward the suspended film/Woltersdorff equations for all frequencies). Although it is shown by calculation ${ }^{43}$ that the (quarter-wave resonance) absorption can be greater than $50 \%$ if the $\mathrm{THz}$ beam is incident on the substrate side first (Supplementary Note 11), this geometry does not easily allow for use as a transmission-mode modulator, because changes in the sheet resistance have less effect on the transmittance compared to the geometry employed in our experiments. Nevertheless, it could still be useful as a reflectionmode device, but the major challenge of engineering low sheet resistance values remains, since absorption greater than $80 \%$ in this geometry requires sheet resistance values of less than $100 \Omega /$ $\square$.

We were able to achieve a large transmission DoM by improving the transconductance (allowing the sheet resistance of a single device to span a broad range) of the graphene on ODTS SAM layer. The high-quality, large-domain graphene films on SAM used here were confirmed via Raman mapping ${ }^{44,} 45$ (Supplementary Note 8), and suggest that substrate modification improves the sheet resistance by improving mobility at high carrier density ${ }^{28,}{ }^{6}$ (Supplementary Fig. 15). Although other materials can exhibit tunable conductance such as vanadium dioxide $\left(\mathrm{VO}_{2}\right)^{47-49}, \mathrm{NbN}^{37-39}, \mathrm{ITO}^{40}$, metal thin films or even multilayer graphite, the conductance of monolayer graphene can be easily tuned via external voltages due to the extremely high 
mobility. For high-frequency applications, the mobility of singlelayer graphene is superior to bilayer (multilayer) graphene ${ }^{50}$, and other semiconducting materials ${ }^{42,51}$, but fabrication remains a challenge for large-area devices which precludes electronically tunable devices operating at very low sheet resistance (even in the simple case of EM incidence on the substrate side first). A combination of substrate modification to improve mobility with techniques such as electrolyte gating ${ }^{33}$ or device integration ${ }^{17}$ could yield electrically controlled devices with high modulation frequency and low operation voltage (see Supplementary Note 7). Additional improvements in large-area (mm-scale) graphene synthesis and transfer will foster better control from the transmission-to-absorption regimes (towards the reflectiondominating regime), as recent reports on large-area CVD graphene demonstrate mobility values as large as 350,000 $\left(\mathrm{cm}^{2} \mathrm{~V}^{-1} \mathrm{~s}\right.$ ${ }^{-1}$ ) with sheet resistance spanning $\sim\left(e^{2} / 4 \hbar\right)$ to $500\left(e^{2} / 4 \hbar\right)$ at DC applying only $\sim 5 V_{\mathrm{g}}^{52}$. Our studies reveal the opportunity for a device based on the tunable electronic properties, without complicated fabrication steps required for other $\mathrm{THz}$ modulation schemes such as metamaterials $29,30,53$, nanophotonic resonators $^{54}$, thin film thickness tuning ${ }^{37-40,47}$ or materials with switchable metal-to-insulator phase transitions ${ }^{47-49}$.

We have demonstrated that the control of the large-area graphene DC sheet resistance enables the tuning, either electrically or chemically, from the transmission to absorption-dominating regimes at $\mathrm{mm}$ wave to $\mathrm{THz}$ frequencies. This could have wideranging application in the tunable and controllable manipulation of $\mathrm{mm}$ wave and $\mathrm{THz}$ light, including, for example, gate tunable $\mathrm{THz}$ modulators, electronically steerable phased array antennas and radar and tunable variable focus lenses and cones. However, while we have clearly demonstrated a single layer tuned from the transmission to absorption regimes (the gray shaded region in Fig. 4c where $A>T, R$ ), we have not pushed well below the inflection point between poor to excellent conductor (the lower bound in Fig. 4c where $R>T, A$ ). Future work with increased conductivity (e.g., by improved materials synthesis, using other two-dimensional materials other than graphene, etc) may enable complete tuning from strong transmitter, to strong absorber, to strong reflector, and would enable new technologies to manipulate EM radiation in powerful ways.

\section{Methods \\ CVD graphene growth. Fully continuous monolayer graphene films are grown on copper foils (Alfa Aesar 94555) via chemical vapor deposition (CVD) in a 5-inch- diameter quartz tube. Large-domain ( $\mathrm{mm}$-scale grain boundaries) graphene films are synthesized using a fast, oxygen-assisted growth using two stages of methane flow ( 0.8 and $2.4 \mathrm{sccm}$ flow rates) to separate nucleation and edge growth as presented in ref. ${ }^{27}$. All gases are left flowing until the furnace cools to less than 300 ${ }^{\circ} \mathrm{C}$. The entire CVD process occurs with the quartz tube evacuated by a dry scroll pump.}

ODTS SAM deposition. High-resistivity $\mathrm{Si}$ wafers with a $90-\mathrm{nm}$-thick, gate-quality $\mathrm{SiO}_{2}$ layer on top are cleaned in hot piranha solution for $1 \mathrm{~h}$ followed by cleaning in deionized (DI) water. The wafers are placed in a vacuum chamber with $50 \mu \mathrm{L}$ of ODTS solution in a glass vial. The chamber is then pumped down and the ODTS is allowed to self-assemble on the $\mathrm{SiO}_{2}$ surface for $6 \mathrm{~h}$.

Graphene transfer. Graphene covering a copper foil $\left(\approx 1 \mathrm{~cm}^{2}\right)$ is coated with poly (methyl methacrylate) (PMMA) solution and etched in $5 \%$ ammonium persulfate (APS) solution. The floating PMMA/graphene stack is washed in DI water and wet transferred onto the substrate. For BI doping experiments, BI $(0.03 \mathrm{M})$ was dissolved in a solution of APS, $\mathrm{H}_{2} \mathrm{SO}_{4}$ and $\mathrm{H}_{2} \mathrm{O}_{2}$ for copper etching. This process simultaneously etches the copper and dopes the graphene as described in ref. ${ }^{31}$ Following transfer, the wafer is dried overnight. Before PMMA removal, the wafer is heated on a hotplate at $130 \mathrm{C}$ for $30 \mathrm{~min}$. After cooling to room temperature, the wafer is placed in an acetone bath for $24 \mathrm{~h}$ for PMMA removal. The wafers are finally rinsed with isopropyl alcohol, and dried with $\mathrm{N}_{2}$ gas.

Electrical characterization of graphene devices. To probe the electrical properties of the transferred graphene films, four $\mathrm{Cr} / \mathrm{Au}(20 / 50 \mathrm{~nm})$ electrodes were deposited by electron beam deposition using a shadow mask. All devices use the same shadow mask to maintain a consistent area to be measured. The electrodes were deposited in a square geometry with $\approx 5 \mathrm{~mm}$ gap separation between each electrode. The current between two diagonally opposed electrodes, representing the drain and source electrodes, was measured with $100 \mathrm{mV}$ bias applied. Gate voltages were applied through the $\mathrm{SiO}_{2}$ layer, with sweeps between -7 and $45 \mathrm{~V}$ (experimentally determined window to avoid leakage). Sheet resistance measurements were performed using a lock-in amplifier and a 4-point van der Pauw method.

THz characterization. An Emcore PB7200 broadband frequency-domain photomixing spectrometer was used to perform $\mathrm{THz}$ transmission measurements through the graphene-on-Si etalon structures. As shown in Supplementary Fig. 1, one photomixer acts as a $\mathrm{THz}$ transmitter, and a separate photomixer as a $\mathrm{THz}$ receiver; one of two distributed feedback lasers is tuned by temperature to perform the broadband difference frequency sweep between 0.2 and $1.2 \mathrm{THz}$ with a 500 $\mathrm{MHz}$ step $^{24}$. An aperture was placed between the sample and the THz beam to ensure that the $\mathrm{THz}$ beam area $(\approx 3 \mathrm{~mm}$ diameter) was consistent from sample to sample, and less in area than the graphene film. The THz beam was then aligned at the geometric center of the four electrodes on the graphene film.

Theoretical calculations. The trend lines according to Eqs. 1 and 2 in Fig. $1 \mathrm{~b}$ are plotted using a linear variation in $\tau$, and $E_{\mathrm{F}}$ from $50 \mathrm{fs}$, and $50 \mathrm{meV}$, to $200 \mathrm{fs}$ and $200 \mathrm{meV}$ (with a step size of $25 \mathrm{fs}$ and $25 \mathrm{meV}$ ), respectively. Figure $1 \mathrm{c}$, d plots the conductance value at $100 \mathrm{GHz}$ varying $\tau$, and $E_{\mathrm{F}}$ from $0.5 \mathrm{fs}$, and $0.5 \mathrm{meV}$, to $200 \mathrm{fs}$ and $600 \mathrm{meV}$ (with a step size of $0.5 \mathrm{fs}$ and $0.5 \mathrm{meV}$ ), respectively.

The transmittance vs. frequency plots for various sheet resistances (dotted lines) in Fig. $3 c-h$ were calculated using the transmission line model as in ref. ${ }^{40}$. The model assumes quasi-plane wave radiation, substrate index of refraction $n=3.41$ and the graphene sheet is treated as a shunt, lumped impedance, $Z_{\mathrm{g}}$, where the real part of the graphene impedance (admittance) is equal to the measured DC resistance (conductance), assuming negligible imaginary contributions. The substrate thickness was inputted accordingly during calculation, and variations from device to device are listed in Supplementary Note 12.

The fitting of measured transmittance data follows our previous work as described in ref. ${ }^{9}$. To calculate the AC conductance data points (Fig. 4a, b) the transmission matrix method was performed using a least-mean-squares fit procedure (Supplementary Note 5). For each data point, one transmission peak and two neighboring valleys were used to calculate the admittance (Supplementary Note 13). The thickness of the substrate was varied accordingly for each device (Supplementary Note 12).

The fitting for trend lines in Fig. 4a, b use Eqs. 1 and 2 and a least-meanssquares fitting procedure consisting of the real part of the calculated AC conductance values, and the measured DC value to estimate $E_{\mathrm{F}}$ and $\tau$ for each device. Up to the final fitting procedure (to estimate $E_{\mathrm{F}}$ and $\tau$ ), all data fitting consisted of both real and imaginary contributions.

Data availability. The data that support the findings of this study are available from the corresponding author upon request.

Received: 12 July 2017 Accepted: 22 November 2017

Published online: 20 December 2017

\section{References}

1. Fiori, G. et al. Electronics based on two-dimensional materials. Nat Nanotechnol. 9, 768-779 (2014).

2. Chhowalla, M. et al. The chemistry of two-dimensional layered transition metal dichalcogenide nanosheets. Nat. Chem. 5, 263-275 (2013).

3. Butler, S. Z. et al Progress, challenges, and opportunities in two-dimensional materials beyond graphene. ACS Nano 7, 2898-2926 (2013).

4. Bonaccorso, F., Sun, Z., Hasan, T. \& Ferrari, A. C. Graphene photonics and optoelectronics. Nat. Photonics 4, 611-622 (2010).

5. Koppens, F. H. L. et al. Photodetectors based on graphene, other twodimensional materials and hybrid systems. Nat. Nanotechnol. 9, 780-793 (2014).

6. Yan, H. et al. Infrared spectroscopy of wafer-scale graphene. ACS Nano 5, 9854-9860 (2011).

7. Horng, J. et al. Drude conductivity of Dirac fermions in graphene. Phys. Rev. B 83, 165113 (2011).

8. Rouhi, N. et al. Terahertz graphene optics. Nano Res. 5, 667-678 (2012).

9. Zhang, W., Pham, P. H. Q., Brown, E. R. \& Burke, P. J. AC conductivity parameters of graphene derived from $\mathrm{THz}$ etalon transmittance. Nanoscale $\mathbf{6}$, 13895-13899 (2014).

10. Sensale-Rodriguez, B. et al. Broadband graphene terahertz modulators enabled by intraband transitions. Nat. Commun. 3, 780 (2012). 
11. Sensale-Rodriguez, B. et al. Extraordinary control of terahertz beam reflectance in graphene electro-absorption modulators. Nano Lett. 21, 936-941 (2012).

12. Ren, L. et al. Terahertz and infrared spectroscopy of gated large-area graphene. Nano Lett. 12, 3711-3715 (2012).

13. Cervetti, C. et al. Sub-terahertz frequency-domain spectroscopy reveals singlegrain mobility and scatter influence of large-area graphene. Adv. Mater. 27, 2635-2641 (2015)

14. Min Woo, J., Kim, M.-S., Woong Kim, H. \& Jang, J.-H. Graphene based salisbury screen for terahertz absorber. Appl. Phys. Lett. 104, 81106 (2014).

15. Maeng, I. et al. Gate-controlled nonlinear conductivity of dirac fermion in graphene field-effect transistors measured by terahertz time-domain spectroscopy. Nano Lett. 12, 551-555 (2012).

16. Buron, J. D. et al. Graphene mobility mapping. Sci. Rep. 5, 12305 (2015).

17. Liang, G. et al. Integrated terahertz graphene modulator with $100 \%$ modulation depth. ACS Photonics 2, 1559-1566 (2015).

18. Mics, Z. et al. Thermodynamic picture of ultrafast charge transport in graphene. Nat. Commun. 6, 7655 (2015).

19. Woltersdorff, W. Uber die optischen Konstanten dunner Metallschichten im langwelligen Ultrarot. Z. fur Phys. 91, 230-252 (1934).

20. Sten, J. C. E. \& Koivisto, P. K. Optimum transparent absorbers of electromagnetic waves. IEEE Trans. Electromagn. Compat. 50, 1011-1014 (2008).

21. Nair, R. R. et al. Fine structure constant defines visual transparency of graphene. Science 320, 1308 (2008).

22. Gusynin, V. P., Sharapov, S. G. \& Carbotte, J. P. On the universal ac optical background in graphene. New J. Phys. 11, 95013 (2009).

23. Falkovsky, L. A. Optical properties of graphene and IV-VI semiconductors. Phys.-Uspekhi 51, 887 (2008).

24. Demers, J. R., Logan, R. T. \& Brown, E. R. An optically integrated coherent frequency-domain $\mathrm{THz}$ spectrometer with signal-to-noise ratio up to $80 \mathrm{~dB}$. In 2007 International Topical Meeting on Microwave Photonics (Ed. Darcie, T.E.) 92-95 (IEEE, 2007).

25. Li, X. et al. Large-area synthesis of high-quality and uniform graphene films on copper foils. Science 324, 1312-1314 (2009)

26. Suk, J. W. et al. Transfer of CVD-grown monolayer graphene onto arbitrary substrates. ACS Nano 5, 6916-6924 (2011).

27. Pham, P. H. Q. et al. Controlling nucleation density while simultaneously promoting edge growth using oxygen-assisted fast synthesis of isolated largedomain graphene. Chem. Mater. 28, 6511-6519 (2016).

28. Lee, W. H. et al. Control of graphene field-effect transistors by interfacial hydrophobic self-assembled monolayers. Adv. Mater. 23, 3460-3464 (2011).

29. Gao, W. et al. High-contrast terahertz wave modulation by gated graphene enhanced by extraordinary transmission through ring apertures. Nano. Lett. 14, 1242-1248 (2014).

30. Fang, Z. et al. Active tunable absorption enhancement with graphene nanodisk arrays. Nano. Lett. 14, 299-304 (2014).

31. Kim, S. J. et al. Simultaneous etching and doping by $\mathrm{Cu}$-stabilizing agent for high-performance graphene-based transparent electrodes. Chem. Mater. 26, 2332-2336 (2014).

32. Batrakov, K. et al. Enhanced microwave-to-terahertz absorption in graphene. Appl. Phys. Lett. 108, 123101 (2016).

33. Balci, O., Polat, E. O., Kakenov, N. \& Kocabas, C. Graphene-enabled electrically switchable radar-absorbing surfaces. Nat. Commun. 6, 6628 (2015).

34. Wu, B. et al. Experimental demonstration of a transparent graphene millimetre wave absorber with $28 \%$ fractional bandwidth at $140 \mathrm{GHz}$. Sci. Rep. 4, 4130 (2014).

35. Grande, M. et al. Optically transparent microwave polarizer based on quasimetallic graphene. Sci. Rep. 5, 17083 (2015)

36. Thongrattanasiri, S., Koppens, F. H. L. \& Garcia De Abajo, F. J. Complete optical absorption in periodically patterned graphene. Phys. Rev. Lett. 108 47401 (2012).

37. Gorshunov, B. P., Fedorov, I. V., Kozlov, G. V., Volkov, A. A. \& Semenov, A. D. Dynamic conductivity and the coherence peak in the submillimeter spectra of superconducting NbN films. Solid State Commun. 87, 17-21 (1993).

38. Gorshunov, B. P. et al. Measurement of electrodynamic parameters of superconducting films in the far-infrared and submillimeter frequency ranges. Int. J. Infrared Millim. Waves 14, 683-702 (1993).

39. Pronin, A. V. et al. Direct observation of the superconducting energy gap developing in the conductivity spectra of niobium. Phys. Rev. B 57, 14416-14421 (1998).

40. Brown, E. R., Zhang, W. D., Chen, H. \& Mearini, G. T. THz behavior of indium-tin-oxide films on p-Si substrates. Appl. Phys. Lett. 107, 91102 (2015).

41. Sensale-Rodriguez, B. et al. Exceptional tunability of THz reflectance in graphene structures. In 2012 37th International Conference on Infrared, Millimeter, and Terahertz Waves (Eds Lewis, R. A. \& Zhang, C.) 1-3 (IEEE, 2012)
42. Sensale-Rodriguez, B. et al. Unique prospects for graphene-based terahertz modulators. Appl. Phys. Lett. 99, 113104 (2011).

43. Zanotto, S. et al. Coherent absorption of light by graphene and other optically conducting surfaces in realistic on-substrate configurations. APL Photonics 2, 16101 (2017).

44. Ferrari, A. C. et al. Raman spectrum of graphene and graphene layers. Phys. Rev. Lett. 97, 187401 (2006).

45. Das, A. et al. Monitoring dopants by Raman scattering in an electrochemically top-gated graphene transistor. Nat. Nanotechnol. 3, 210-215 (2008).

46. Rakheja, S., Kumar, V. \& Naeemi, A. Evaluation of the potential performance of graphene nanoribbons as on-chip interconnects. Proc. IEEE 101, 1740-1765 (2013).

47. Dressel, M. \& Gruner, G. Electrodynamics of Solids: Optical Properties of Electrons in Matter (Cambridge University Press, Cambridge, 2002).

48. Verleur, H. W., Barker, A. S. \& Berglund, C. N. Optical properties of VO2 between 0.25 and $5 \mathrm{eV}$. Rev. Mod. Phys. 172, 788 (1968).

49. Liu, M. et al. Terahertz-field-induced insulator-to-metal transition in vanadium dioxide metamaterial. Nature 487, 345-8 (2012).

50. Dean, C. R. et al. Boron nitride substrates for high-quality graphene electronics. Nat. Nanotechnol. 5, 722-726 (2010).

51. Wang, L. One-dimensional electrical contact to a two-dimensional material. Science 342, 614-617 (2013).

52. Banszerus, L. et al. Ultrahigh-mobility graphene devices from chemical vapor deposition on reusable copper. Sci. Adv. 1, e1500222 (2015).

53. Rahm, M., Li, J. S. \& Padilla, W. J. THz wave modulators: a brief review on different modulation techniques. J. Infrared Millim. Terahertz Waves 34, 1-27 (2013).

54. Majumdar, A., Kim, J., Vuckovic, J. \& Wang, F. Graphene for tunable nanophotonic resonators. IEEE J. Sel. Top. Quantum Electron 20, 68-71 (2014).

\section{Acknowledgements}

We appreciate the conversations with D. Fishman, J. Hes and D. Humphrey. SEM and oxygen plasma work was performed at the Irvine Materials Research Institute (IMRI). Raman spectroscopy work was performed at the UC Irvine Laser Spectroscopy Facility (LSF). CVD synthesis and e-beam evaporation was performed at the Integrated Nanosystems Research Facility (INRF). This work was funded by the Army Research Office through the ARO-MURI Program and an ARO-Core Grant (Contract No: W911NF-111-0024 and W911NF-09-1-0319).

\section{Author contributions}

P.H.Q.P., W. Zhang, E.R.B. and P.J.B. conceived the experiments. P.H.Q.P., N.V.Q., W. Zhou and D.S. fabricated the devices. P.H.Q.P., N.V.Q. and W. Zhang performed material characterization and THz measurements. P.H.Q.P., W. Zhang, J.L., E.R.B. and P. J.B. performed theoretical calculations and fitting. P.H.Q.P., W. Zhang, E.R.B. and P.J.B. wrote the manuscript.

\section{Additional information}

Supplementary Information accompanies this paper at https://doi.org/10.1038/s41467 017-02336-z.

Competing interests: The authors declare no competing financial interest.

Reprints and permission information is available online at http://npg.nature.com/ reprintsandpermissions/

Publisher's note: Springer Nature remains neutral with regard to jurisdictional claims in published maps and institutional affiliations.

Open Access This article is licensed under a Creative Common Attribution 4.0 International License, which permits use, sharing, adaptation, distribution and reproduction in any medium or format, as long as you give appropriate credit to the original author(s) and the source, provide a link to the Creative Commons license, and indicate if changes were made. The images or other third party material in this article are included in the article's Creative Commons license, unless indicated otherwise in a credit line to the material. If material is not included in the article's Creative Commons license and your intended use is not permitted by statutory regulation or exceeds the permitted use, you will need to obtain permission directly from the copyright holder. To view a copy of this license, visit http://creativecommons.org/ licenses/by/4.0/

(C) The Author(s) 2017 